\title{
Application of ionospheric tomography for high frequency oblique incidence ray tracing
}

\author{
Sandip Nandi ${ }^{1{ }^{\star}}$, Bijoy Bandyopadhyay ${ }^{2}$ \\ ${ }^{1}$ Kalyani Govt. Engineering College, Dept. of Electronics and Communication Engineering, Kalyani, West Bengal, India \\ ${ }^{2}$ Institute of Radio Physics and Electronics, University of Calcutta, Kolkata, India
}

\section{Article history}

Received June 23, 2015; accepted November 27, 2015.

Subject classification:

Ionosphere, Radiowave propagation, Ray tracing, Tomography.

\begin{abstract}
Recognizing the applicability of tomographic imaging in the ionosphere, the reconstruction method has been applied to generate more realistic low latitude ionospheric background based on the Ionex TEC data and also direct measurement TEC data over West Bengal, India. Using these backgrounds, ray tracing of high frequency waves has been performed. Influence of Earth's magnetic field and also the existence of horizontal gradient of electron concentration in the ionosphere are considered separately for analyzing the ray tracing algorithm. Evaluation of ray tracing parameters and maximum usable frequency for different ground range ensures the possibility of application of ionospheric tomography technique for accurate ray tracing. The analysis shows accurate ray tracing for HF waves, thus defeating the restriction of choice of ionospheric model for analytical ray tracing methods. Performance analysis of the ray tracing technique presented in this paper ensures the consistency of speed with the other methods.
\end{abstract}

\section{Introduction}

From the early days of radio communications, long distance transionospheric communication with high frequency (HF) radio waves has been used extensively. The ionospheric effects on transionospheric signals include absorption, refraction, reflection, phase and group delay, frequency shift, polarization shift, and Faraday rotation. Most of these effects are related to the varying refractive index of the ionosphere, which can be directly linked to the free electron content. Thus, the ionospheric medium causes perturbation of HF signals passing through it. Hence, the performance of some communication systems, e.g. HF broadcasting, over-the-horizon radar systems and direction finding systems critically depends on accurate ray tracing through a realistic ionospheric model.

Research of HF radio wave propagation through ionosphere started earlier based on geometrical optic theory [Poeverline 1948, Poeverline 1949, Hines 1951,
Haselgrove 1955] and full wave theory [Försterling 1942, Rydbeck 1944, Budden 1952, Budden 1988, Davies 1990]. Since then, two main types of ray tracing have been developed. First, the analytical ray tracing [Anderson et al. 1989, Bennett et al. 2004] and second, numerical technique [Jones 1966, Reilly 1991]. The ray tracing can be efficiently developed using the estimated parameters of ground range, reflection height, phase path, and group path. Some of the important works on ray tracing are found in Coleman [1998], Jones and Stephenson [1975], and Nickish [2008]. Norman and Cannon [1997, 1999] introduce SMART for analytical ray tracing using quasi-parabolic segmentation of the ionosphere and including the horizontal gradient. This method offers a ten to hundredfold speed improvement over the numerical methods with slight loss of accuracy. In this respect, analytical ray tracing techniques are much faster than numerical approaches, but are restricted in the ionospheric models to which they can be applied. Instead, Azzarone et al. [2012] introduce a windows software tool, namely IONORT, for implementing a 3-dimensional (3-D) ray tracing of high frequency waves in the ionosphere. This 3-D numerical ionospheric profile representation is based on joint utilization of F2 layer peak critical frequency $\left(\mathrm{f}_{0} \mathrm{~F}_{2}\right)$, propagation factor $\left(\mathrm{M}(3000) \mathrm{F}_{2}\right)$, electron density profile from two reference ionospheric stations, and global (IRI) ionospheric models. The ray tracing algorithm is based on the numerical (Runge-Kutta or Adams-Bushford predictor) solution of system of differential equations, i.e. 'system of non-linear equations' with Hamiltonian formulation. In continuation, Settimi et al. [2013] discuss the application of IONORT on different electron density grids for synthesizing oblique ionograms. The comparison of IONORT with measured oblique ionograms demonstrates the reliability of the software. However, 
Settimi et al. [2013] algorithm tried to solve a system of non-linear equations, but not considering the effect of horizontal gradient.

For radio systems applications, choice of realistic ionospheric model that describe accurate electron density specification is very important for the ionospheric ray tracing. Ionospheric tomography may provide the highaccuracy electron density specification. Recent developments of computerized ionospheric tomography (CIT) [Andreeva et al. 1990, Kunitsyn and Tereshchenko 1992, Kunitake et al. 1995, Markkanen et al. 1995, Mitchell et al. 1995, Pryse et al. 1995, Huang et al. 1998] lead the researchers to image electron density profile for the ionosphere. CIT is a pixel based method of imaging the ionosphere by solving system of linear equations with a priori knowledge of the total electron content (TEC, integrated electron density) along ray paths. This method reconstructs a two dimensional electron density profile of the ionosphere. Some recent works are given by Kersley et al. [1997] and Pryse et al. [1998] and a wider review by Leitinger [1999]. These works indicate that tomographic imaging will provide a clear vision of the electron density distribution in the ionosphere.

In this paper, a two dimensional ionospheric electron density profile has been reconstructed by CIT technique. Different algorithms are available for the reconstruction, e.g. algebraic reconstruction technique (ART), simultaneous ART (SART), multiplicative ART (MART), etc. Among them, performance of SART for reconstruction is better compared to ART and simultaneous iterative reconstruction technique (SIRT) in respect of statistical errors and computational load irrespective of the geometry [Nandi and Bandyopadhyay 2015], and is used in this paper to image the ionosphere. The TEC data obtained from the networks of global positioning system (GPS) receivers located around the Earth are directly used to retrieve the information of the structure of ionosphere. The reconstruction technique is briefly described in Section 2.

It is noteworthy that, TEC can be represented as the line integral of the electron density along a ray trajectory, usually vertical from the surface of the Earth to a particular height in the ionosphere (VTEC) or lineof-sight from the satellite to the receiver (slant TEC). The ionospheric delay is a major source of error for single frequency operation. Signals are perturbed in amplitude, phase and polarization during its travel through the ionosphere. Specifically, change of phase due to refraction leads to propagation errors typically up to $50 \mathrm{~m}$ for single-frequency GPS users [Jakowski et al. 1996]. If dual frequency measurements are considered, firstorder range error can be determined and removed. The ionospheric refraction cannot be ignored at these fre- quencies. For single-frequency operation, ionosphere induced propagation errors up to $50 \mathrm{~m}$ have to be considered depending on the TEC along the ray path. Thus, the calibration of TEC is crucial in tomographic applications. The calibration of data is briefly described in Section 3.

Next, using the reconstructed ionosphere as background, the ray tracing for radio waves has been performed under isotropic and homogeneous conditions. Comparisons of ray tracing parameters are also established under the presence of Earth's magnetic field and also under the presence of horizontal gradients of electron concentration in the ionosphere. A comparative study of the maximum usable frequency (MUF) and ray trace parameters reveals the efficiency of the ray tracing using tomographic reconstruction. Lastly the entire work has been tested with real observations from GPS receivers in the low latitude region and IRI 2012 model data. The performance analysis of ray tracing with different tomographic model and also comparison with other existing analytical methods reveals that the speed of the ray tracing algorithm presented in this paper is comparable to the analytical methods reported earlier.

\section{Tomographic reconstruction}

The technique of tomographic imaging has been applied for reconstruction of the electron density profile of the ionosphere subject to the advantage that the measurements can be approximated as line-integrals of the electron density. The ray tomography approach projects the ionosphere as a two dimensional cross section and breakup the plane in $\mathrm{N}$ number of pixels, thus transforming the problem into a problem of solving linear system of equations. The basic theory was well established by Austen et al. [1986, 1988], and a good review was given by Raymund [1995]. Considering the advantages of SART [Nandi and Bandyopadhyay 2015], the algorithm has been used in this paper for solving the system of linear equations.

\subsection{Data sources}

The investigation of electron density profile using tomographic technique requires a priori knowledge of vertical total electron content (VTEC) of different latitude and longitude position. For the reconstruction in this paper, the Ionex VTEC data obtained from IGSGPS observation data center (available at http:/ / igscb. jpl.nasa.gov) are used as a priori information. Also the international reference ionosphere (IRI) 2012 model electron density data are used for initial guess values. For the purpose of reconstruction, the ionosphere region is assumed to start from $65 \mathrm{~km}$ altitude and is ex- 
tended up to $1005 \mathrm{~km}$ altitude. The latitude range is chosen from $20^{\circ} \mathrm{N}$ to $40^{\circ} \mathrm{N}$ so as to cover the low latitude region. Entire ionospheric region between the chosen altitude and latitude ranges are divided into small pixels each having vertical dimension of $5 \mathrm{~km}$ and horizontal dimension of $11.12 \mathrm{~km}$ (i.e. $\sim 0.1^{\circ}$ ). This chosen geometry divides the ionospheric region into 37,788 numbers of pixels. The choice of geometry of reconstruction for low latitude region is briefed in Table 1. Nine receiver locations are pointed around the entire latitude range of interest. Since IGS data are obtained for latitude separation of $2.5^{\circ}$ and longitude separation of $5^{\circ}$, the receiver locations are assumed to be separated by $2.5^{\circ}$ in latitude. The longitudes are kept fixed at $85^{\circ} \mathrm{E}$ so as to view the ionosphere as a 2-dimensional region depending on the altitude and latitude only. The coordinates of the receivers are listed in Table 2 at a fixed longitude of $85^{\circ} \mathrm{E}$.

\subsection{SART algorithm}

The linear imaging problem has been solved using SART [Andersen and Kak 1984] and is given by

$$
x_{j}^{k+1}=x_{j}^{k}+\lambda \frac{\sum_{P_{i} \in P_{\varphi}} \frac{P_{i}-\sum_{n=1}^{N} W_{i n} \cdot x_{n}^{k}}{\sum_{n=1}^{N} W_{i n}} \cdot W_{i j}}{\sum_{P_{i} \in P_{\varphi}} W_{i j}}
$$

where $\lambda$ is the relaxation parameter, $x_{j}^{k}$ is the value of the $\mathrm{j}^{\text {th }}$ cell for $\mathrm{k}^{\text {th }}$ iteration, $W_{i j}$ is the weight coefficient of the $j^{\text {th }}$ cell for $\mathrm{i}^{\text {th }}$ ray, and $P_{i}$ is measured ray sum along the $i^{\text {th }}$ ray. Good convergence with small number of iterations can be observed with proper choice of $\lambda$ (typically less than one). The process of iteration first guesses the values of the pixels and then alters these values until it is correct one. All the equations belonging to the same angle at the same time $\left(P_{i} \in P_{\varphi}\right)$ are used before comparing the new values of the pixels with the old one. The advantages of SART are mainly twofold. First, solution of number of unknowns even in high resolution tomographic problems can be found with less computational load. The number of operations in SART is determined by the number of unknowns $(\mathrm{N})$,

\begin{tabular}{lc}
\hline Altitude range & $65-1005 \mathrm{~km}$ \\
Latitude range & $20^{\circ} \mathrm{N}-40^{\circ} \mathrm{N}$ \\
Pixel & 37788 \\
Vertical dimension of one pixel & $5 \mathrm{~km}$ \\
Horizontal dimension of one pixel & $11.12 \mathrm{~km}$ \\
Number of receivers & 9 \\
\hline
\end{tabular}

Table 1. Geometry for the reconstruction.

\begin{tabular}{ccc} 
Receiver No. & Latitude & Longitude \\
\hline 1 & $20^{\circ} \mathrm{N}$ & $85^{\circ} \mathrm{E}$ \\
2 & $22.5^{\circ} \mathrm{N}$ & $85^{\circ} \mathrm{E}$ \\
3 & $25^{\circ} \mathrm{N}$ & $85^{\circ} \mathrm{E}$ \\
4 & $27.5^{\circ} \mathrm{N}$ & $85^{\circ} \mathrm{E}$ \\
5 & $30^{\circ} \mathrm{N}$ & $85^{\circ} \mathrm{E}$ \\
6 & $32.5^{\circ} \mathrm{N}$ & $85^{\circ} \mathrm{E}$ \\
7 & $35^{\circ} \mathrm{N}$ & $85^{\circ} \mathrm{E}$ \\
8 & $37.5^{\circ} \mathrm{N}$ & $85^{\circ} \mathrm{E}$ \\
9 & $40^{\circ} \mathrm{N}$ & $85^{\circ} \mathrm{E}$ \\
\hline
\end{tabular}

Table 2. Site information for 9 GPS receivers.

whereas in non-iterative approaches, number of operations are determined mainly by the size of the design matrix $(\mathrm{N} \times \mathrm{N})$. Secondly SART always iterates to converge to a unique solution irrespective of the situations whether the problem is under determined or over determined. The performance analysis of this method with other iterative methods regarding the convergence and computational load has been already described in Nandi and Bandyopadhyay [2015].

\subsection{Reconstruction of ionosphere}

For the reconstruction of the ionosphere, the data sets for September 1 and 20, 2011, at 10 UT are used. Two dates are chosen for case study under two different ionospheric conditions. On September 1, the ionosphere is considered as quiet, while on September 20, the ionosphere is considered as disturbed due to the post-seismic ionospheric perturbations, since a series of intense earthquake with several aftershock occurred on September 18 and 19, 2011, at Sikkim and Darjeeling areas of India and adjoining Nepal with its epicenter $\left(27.7^{\circ} \mathrm{N}, 88.2^{\circ} \mathrm{E}\right)$ lying near the Sikkim-Nepal border. Seismic activity leads to a deviation in the daily values of F2 layer critical frequency $\left(\mathrm{f}_{0} \mathrm{~F}_{2}\right)$, TEC, and in situ electron density. The IRI 2012 model data for the same day are taken into account as an initial guess. As mentioned earlier, SART algorithm has been used for the reconstruction. Under the reconstruction geometry as detailed in Table 1, the results of reconstruction are given in Figure 1. Figure 1a illustrates the electron density distribution $\left(10^{12} \mathrm{~m}^{-3}\right)$ for IRI 2012 model of the ionosphere on September 20, 2011. The reconstructed image of the ionosphere is shown in Figure $1 b$ for the same date using SART algorithm. The relaxation parameter has been chosen to be less than one $(\lambda<1)$. Comparison of the two figures delineates very good reconstruction of the ionization anomaly, preserving the tilt of the ionosphere as well. Also the crest of the ion- 
ization anomaly is pronounced as reported by Andreeva et al. [2000] and Yeh et al. [2001].

\section{Ray tracing results}

Considering the reconstructed electron density distribution of the ionosphere (as described in Section 2 ) as the background, implementation of the ray tracing algorithm for transmission of radio waves from one point to another on the Earth has been carried out. In the rest of the paper, this reconstructed background is represented as T1 model of the ionosphere. The ionosphere has been segmented into pixels with vertical dimension of $5 \mathrm{~km}$ and horizontal dimension of 11.12 $\mathrm{km}$ (i.e. adopted the reconstruction geometry for the segmentation of the ionosphere). The refractive index (n) for each pixel is obtained by:

$$
n^{2}=1-\frac{X}{1-j Z-\left(\frac{Y_{T}^{2}}{2(1-X-j Z)}\right) \pm \sqrt{\left(\frac{Y_{T}^{4}}{4(1-X-j Z)^{2}}+Y_{L}^{2}\right)}}
$$

where

$$
\begin{gathered}
X=\frac{\omega_{N}^{2}}{\omega^{2}}, Y=\frac{\omega_{B}}{\omega, Y_{L}=\omega_{B} \cos \theta / \omega^{\prime}, Y_{T}=} \\
=\omega_{B} \sin \theta / \omega, Z=V / \omega, \omega_{N}=\left(\frac{N e^{2}}{\varepsilon_{0} m}\right)^{\frac{1}{2}}, \omega_{B}=\frac{B e}{m}
\end{gathered}
$$

Here $\omega_{N}$ is the angular plasma frequency, $\omega_{B}$ is the electron gyro frequency, $v$ is electron collision frequency, $B$ is the intensity of Earth's magnetic field. Assuming the electron plasma to be collision less (i.e. $v \approx 0$ ) Equation (2) becomes

$$
n^{2}=1-\frac{X}{1-\left(\frac{Y_{T}^{2}}{2(1-X)}\right) \pm \sqrt{\left(\frac{Y_{T}^{4}}{4(1-X)^{2}}+Y_{L}^{2}\right)}}
$$

Now Equations (2) and (4) can be used to find out the refractive index for each pixel of the segmented ionosphere. Two refractive indices for $\mathrm{O}$ mode and $\mathrm{X}$ mode are obtained from Equation (2) through the choice of positive or negative signs, which are decided by the Booker's [1935] rule. The Booker's rule states that, to achieve continuity of $n$ for O mode (or X mode), if $\frac{\omega_{C}}{v}>1$, the positive (negative) sign in Equation (4) must be adopted both for $X<1$ and for $X>1$; where $\omega_{C}$ is the critical frequency defined as:

$$
\omega_{C}=\frac{\omega_{B}}{2} \frac{\sin ^{2} \theta}{\cos \theta}
$$

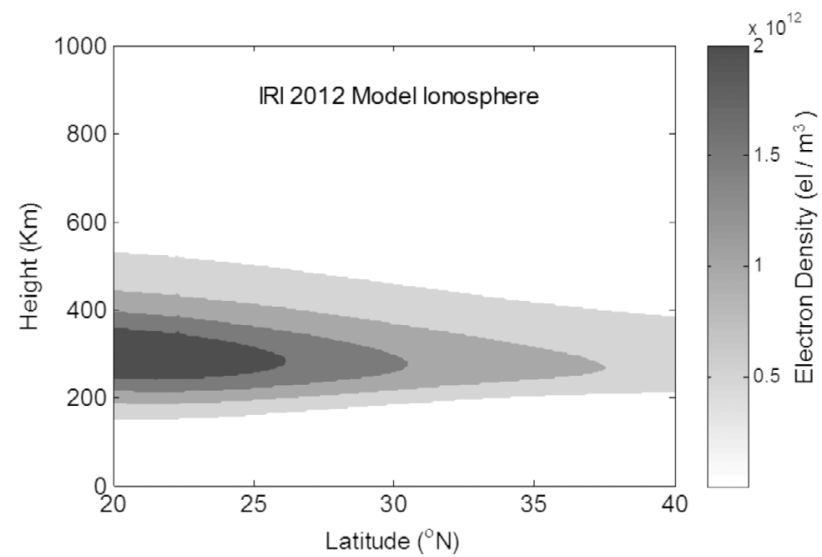

(a)

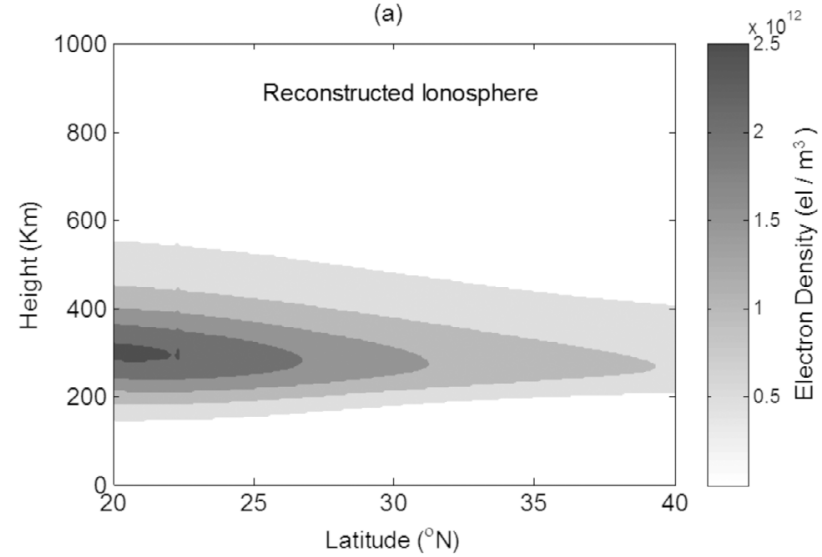

(b)

Figure 1. Electron density $\left(10^{12} \mathrm{~m}^{-3}\right)$ distribution of the ionosphere at 10 UT on September 20, 2011, for: (a) IRI 2012 model (b) reconstruction using SART.

However if $\frac{\omega_{C}}{v}<1$, the positive (negative) sign for $X<1$ and negative (positive) for $X>1$ must be adopted. Since, we have assumed the ionosphere as collision less plasma (i.e., assuming value of $v$ too small that can be neglected), the relation $\frac{\omega_{C}}{v}>1$ holds for this case and we adopt positive (negative) sign in Equation (4) to determine $n$ for $\mathrm{O}$ mode (X mode). Then Snell's law of refraction is applied to find out the ray path for different angle of incidence. The height of the ionosphere is chosen to be located above $65 \mathrm{~km}$ over the ground and radius of the Earth as $6370 \mathrm{~km}$. In the lower atmosphere, the dielectric constant is different than unity by less than 300 parts per million [Bean and Dutton 1966]. So, without any loss of generality, the value of the refractive index below the ionosphere can be assumed as unity. In this paper two distinct cases are considered: Case 1, when the ionosphere is horizontally stratified; and Case 2, when there exists a horizontal gradient of electron concentration in the ionosphere.

\subsection{Case 1: Under horizontal stratification}

When the ionosphere is considered as horizontally stratified, the electron concentration is assumed to be constant in horizontal direction within each vertical 


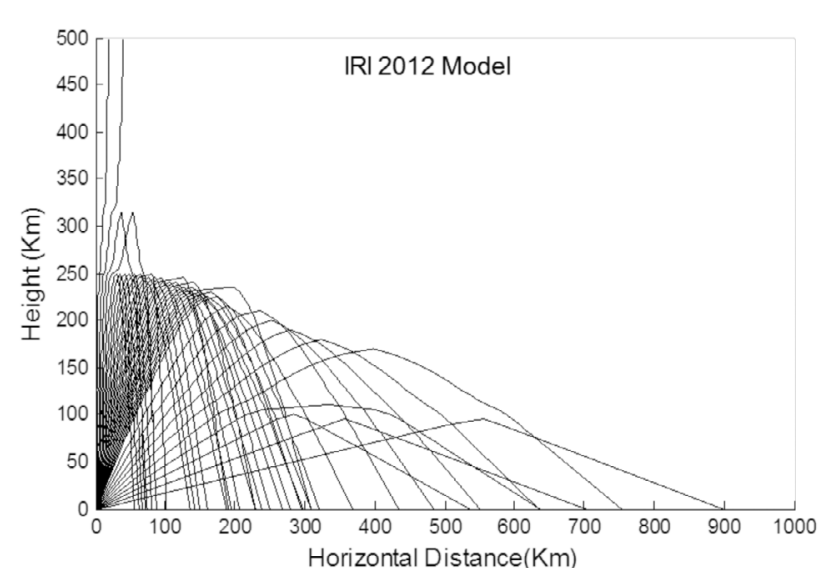

(a)

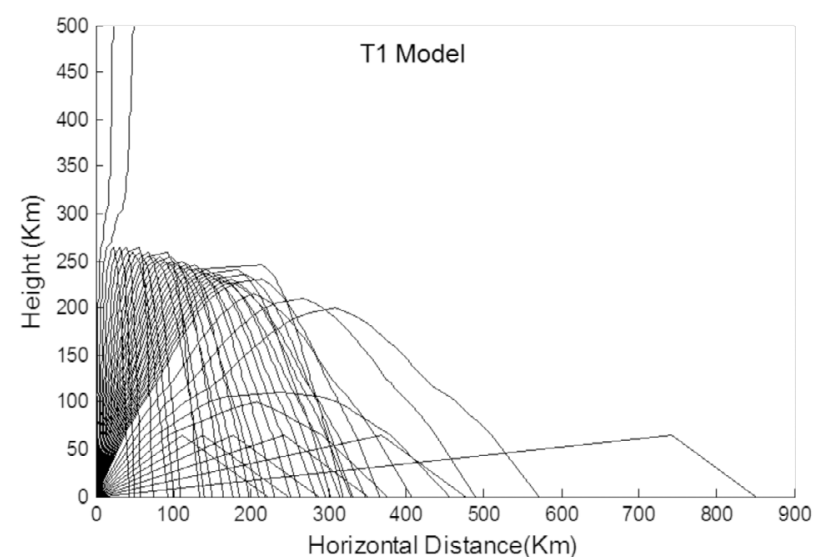

(b)

Figure 2. Ray tracing at 7.5 MHz for different angle of incidence under Case 1 and without Earth's magnetic field when the background is, (a) IRI 2012 model of the ionosphere and (b) T1 model of the ionosphere.

segment. First, neglecting the influence of Earth's magnetic field, i.e. $Y_{L}, Y_{T}=0$ in Equation (4), the refractive index for each segment are obtained. For this purpose, plasma frequency $\left(f_{N}\right)$ is chosen as $3.2 \mathrm{MHz}$ for E layer, 4.6 MHz for F1 layer, and $6.95 \mathrm{MHz}$ for F2 layer. Figure 2 depicts the ray tracing results for different angle of incidence. The incidence angle is varied from $1^{\circ}$ to $30^{\circ}$ in steps of $1^{\circ}$ and then up to $85^{\circ}$ in steps of $5^{\circ}$. The frequency of $7.5 \mathrm{MHz}$ is used for finding the ray paths. The ray tracing results for IRI 2012 model of the ionosphere as background is shown in Figure 2a while for T1 model of the ionosphere as background is shown in Figure 2b.

When Earth's magnetic field is introduced, the situation becomes slightly different as now there exists two modes of propagation: $\mathrm{O}$ mode and $\mathrm{X}$ mode. The values of magnetic field intensity and declination at each of the latitudes for the date of interest are found at NOAA, National Geophysical Data Centre (http:/ / www.ngdc.noaa.gov/geomag/magfield. shtml). Figure 3 describes the ray tracing separately for two background ionosphere under influence of Earth's magnetic field. Clear isolation of $\mathrm{O}$ mode ray (solid line)

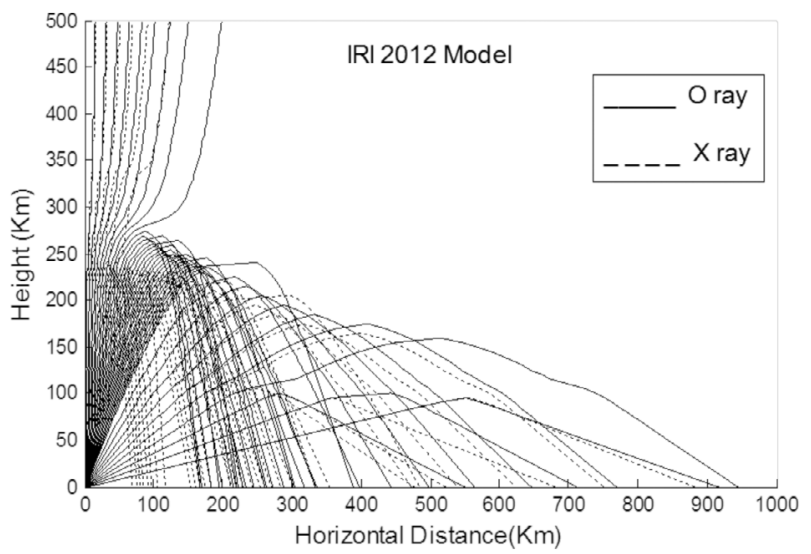

(a)

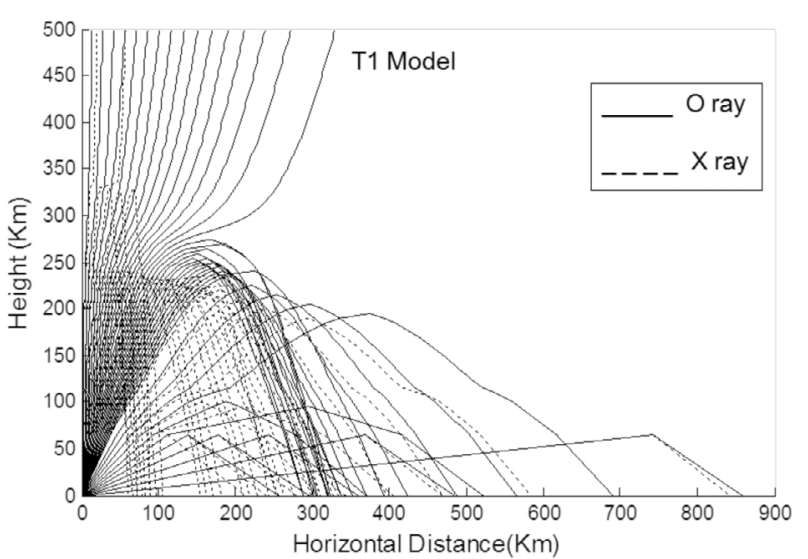

(b)

Figure 3. Ray tracing at 7.5 MHz for different angle of incidence under Case 1 and under influence of Earth's magnetic field when the background is, (a) IRI 2012 model of the ionosphere and (b) T1 model of the ionosphere.

and $\mathrm{X}$ mode ray (dashed line) is observed.

Ray tracing parameters like phase path $(\mathrm{P})$, group path $(\mathrm{P} /)$ and transmission distance or ground range (D) are calculated (in $\mathrm{km}$ ) for different angle of incidence for. Figure 4 depicts the values of these parameters for different angles of incidence under Case 1. Figure 4a describes the parameter values when magnetic field is absent, whereas Figure $4 \mathrm{~b}$ and $4 \mathrm{c}$ describes the parameter values for $\mathrm{O}$ and $\mathrm{X}$ mode propagation respectively, under the influence of magnetic field. Observation from Figure 4 reveals good matching of parameter values for IRI and T1 model of the ionosphere.

\subsection{Case 2: Under horizontal gradient}

When the horizontal gradient of electron concentration exists in the ionosphere, the electron concentration is now assumed to take a fixed value for each pixel of the segmented ionosphere. The same procedures (as described earlier) are followed in this case also. First, neglecting the influence of Earth's magnetic field, the ray tracing using wave frequency of $7.5 \mathrm{MHz}$ are obtained and are shown in Figure 5 with different angles of incidence. Figure $5 \mathrm{a}$ is the ray tracing results con- 


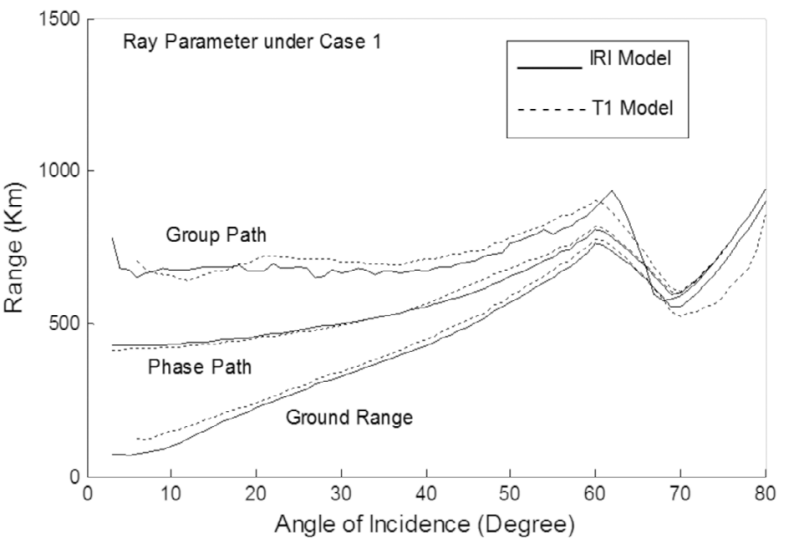

(a)

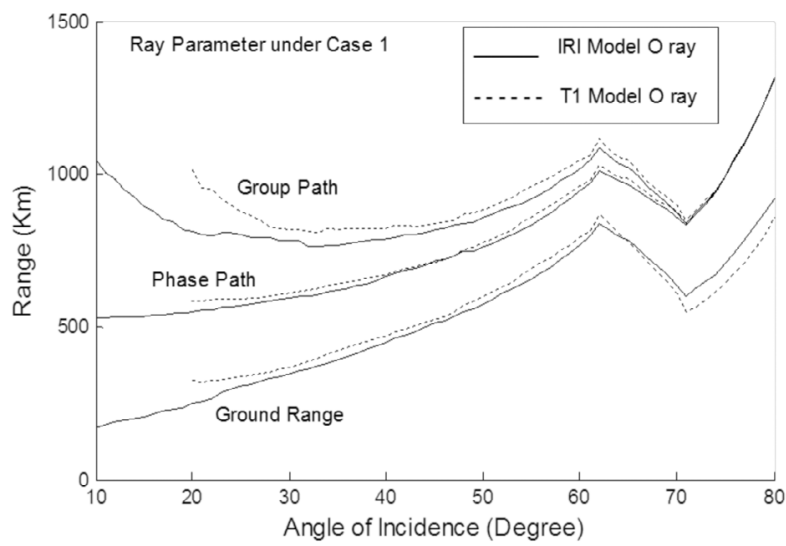

(b)

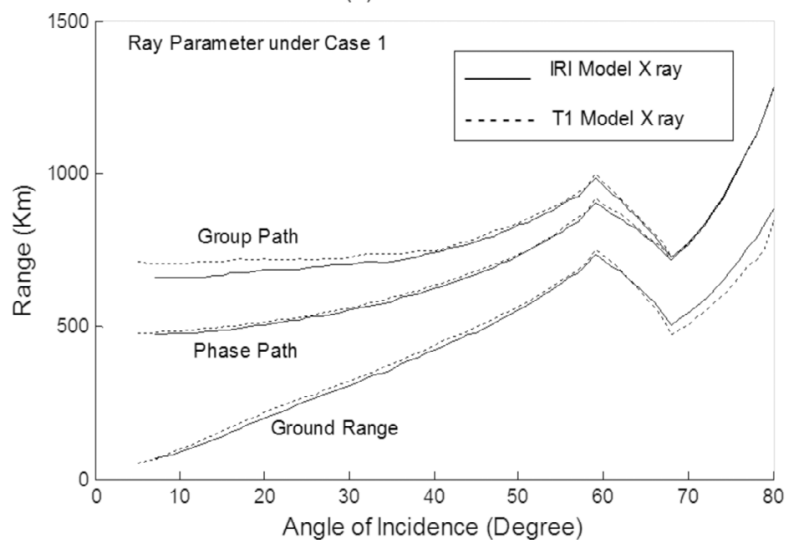

(c)

Figure 4. Ray tracing parameter variation with different angle of incidence under Case 1 at $7.5 \mathrm{MHz}$ for: (a) ray tracing without influence of Earth's magnetic field (b) O mode propagation (c) X mode propagation.

sidering the IRI 2012 model of the ionosphere as background and Figure $5 \mathrm{~b}$ is for the T1 model of the ionosphere. Henceforth considering the presence of Earth's magnetic field, the ray tracing results are shown in Figures $5 \mathrm{c}$ and $5 \mathrm{~d}$ for IRI 2012 model and the T1 model of the ionosphere respectively. Here also O mode and $\mathrm{X}$ mode rays are distinctly observed. The ray tracing parameter variation for this case is shown in Figure 6. Observation shows slight increase of the parameter values compared to the results for Case 1. Calculation of ray parameters shows the expected results that true path is larger than phase path but less than group path.

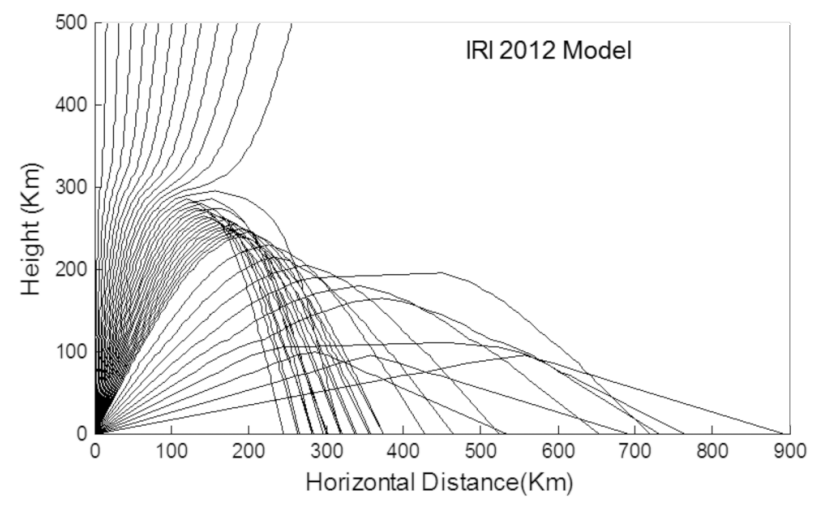

(a)

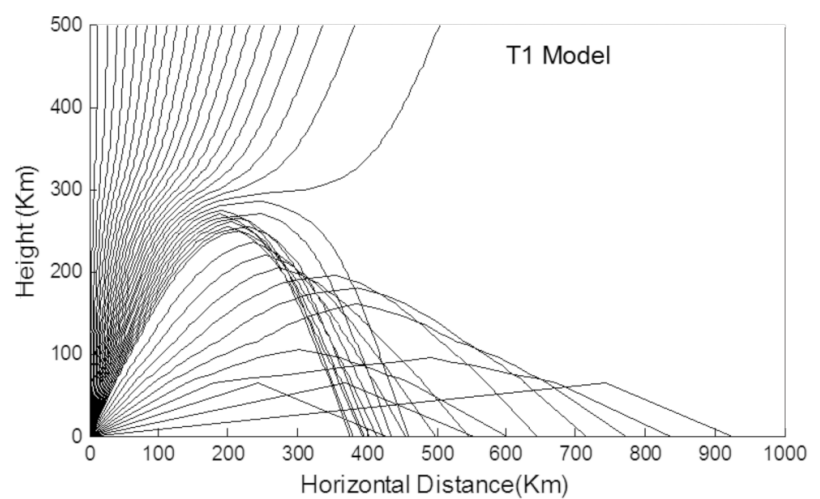

(b)

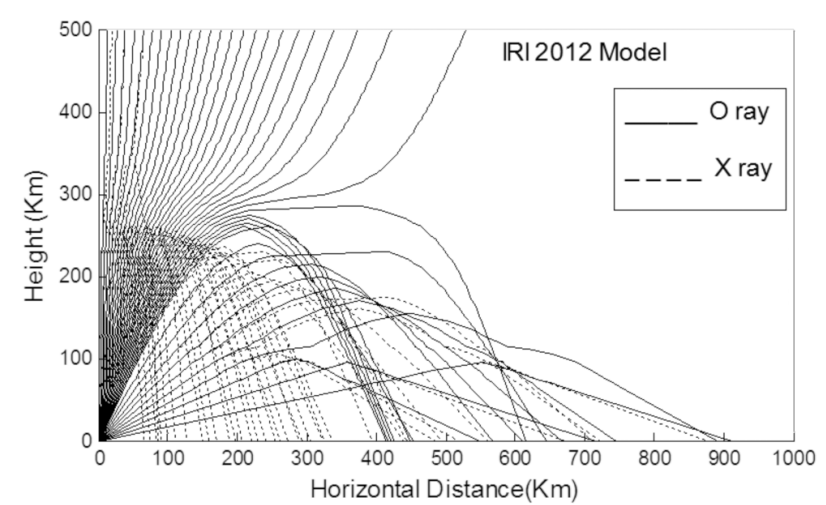

(c)

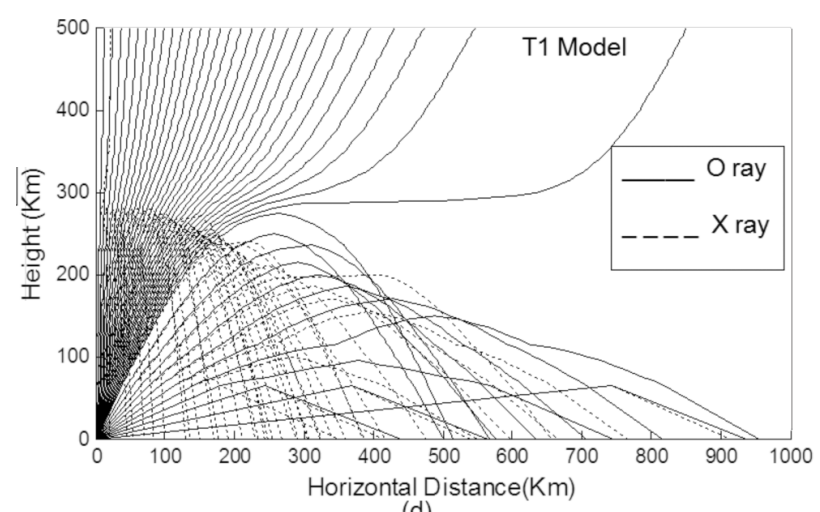

(d)

Figure 5. Ray tracing for different angle of incidence at 7.5 $\mathrm{MHz}$ under Case 2 for: (a) IRI 2012 model of the ionosphere without influence of magnetic field, (b) T1 model of the ionosphere without influence of magnetic field, (c) IRI 2012 model of the ionosphere under influence of magnetic field, (d) T1 model of the ionosphere under influence of magnetic field. 


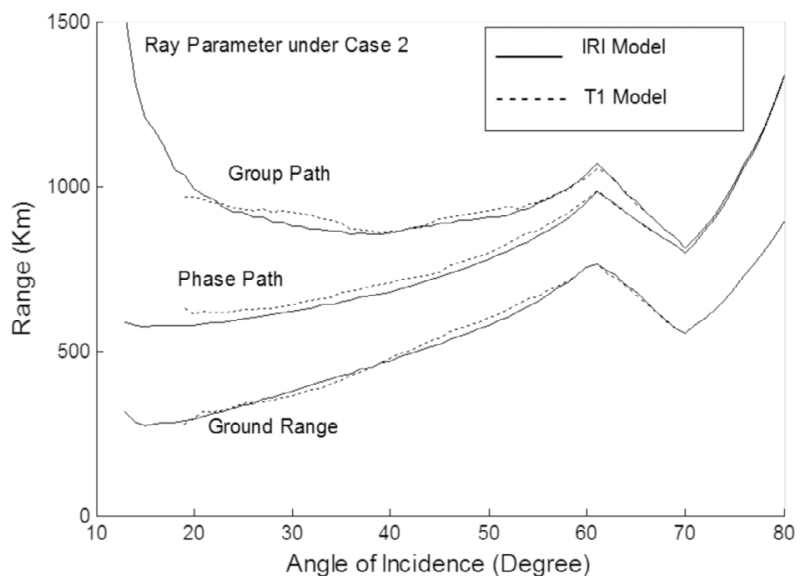

(a)

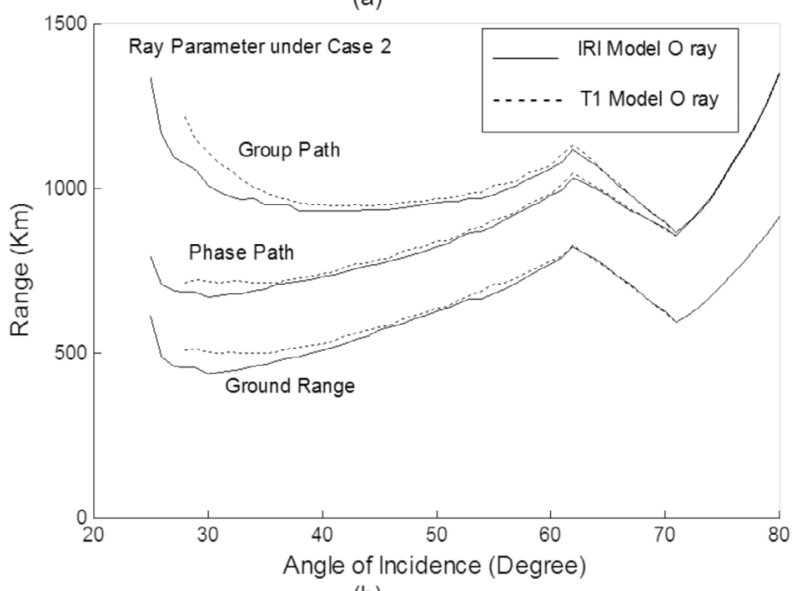

(b)

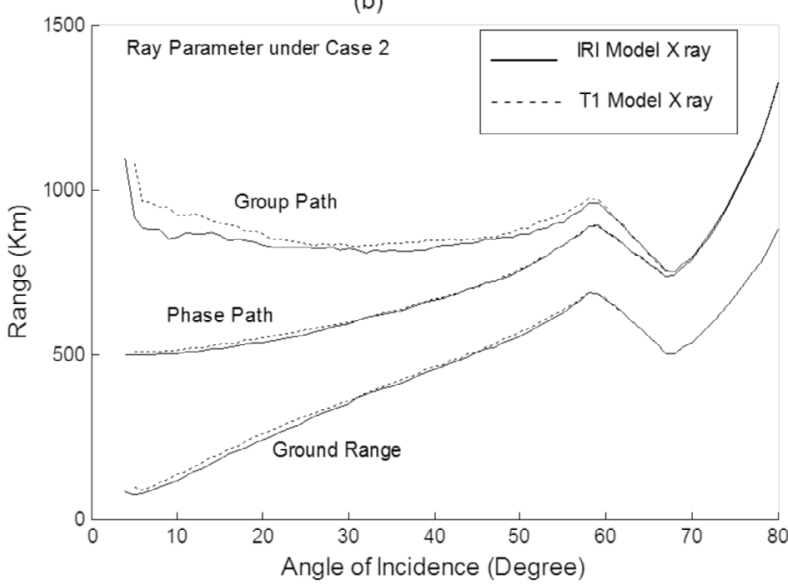

(c)

Figure 6. Ray tracing parameter variation for different angle of incidence at $7.5 \mathrm{MHz}$ under Case 2 for: (a) ray tracing without influence of Earth's magnetic field (b) O mode propagation (c) X mode propagation.

\subsection{Ray tracing with experimental data}

For further analysis and testing, real time TEC data for low latitude ionosphere collected from the Institute of Radio Physics and Electronics of the University of Calcutta, India are used for reconstruction. These TEC values are measured simultaneously from three receivers located at IRPE, Kolkata $\left(22.58^{\circ} \mathrm{N}\right.$, $\left.88.38^{\circ} \mathrm{E}\right), \mathrm{KNC}$, Baharampur $\left(24.09^{\circ} \mathrm{N}, 88.39^{\circ} \mathrm{E}\right)$ and NBU, Siliguri $\left(26.72^{\circ} \mathrm{N}, 88.39^{\circ} \mathrm{E}\right)$. The measured TEC values are calibrated using the following relation:

$$
S T E C_{o b s}=S T E C+B_{S}+B_{R}
$$

where STEC is the slant TEC measured along the path, $B_{S}$ and $B_{R}$ are satellite and receiver biases respectively. Equation (6) indicates that calibration of TEC data requires inclusion of the receiver and satellite interfrequency biases with the measurement. The details about the data and the tomographic reconstruction of the ionosphere based on these data are well described in [Nandi and Bandyopadhyay 2015]. In this paper, three different sets of data are used to reconstruct three new background ionospheres.

Data set 1: The real time TEC data for three receiver locations collected from the Institute of Radio Physics and Electronics of the University of Calcutta, India for September 20, 2011, at 10 UT are used for reconstruction of the ionosphere with the same geometry (as described in Table 1). This reconstructed ionosphere is represented as T2 model of the ionosphere in the rest of the paper and is used as background ionosphere for ray tracing at $7.5 \mathrm{MHz}$.

Data set 2: The IGS data and the real time TEC data for September 20, 2011, at 10 UT are merged together to form a new TEC data set for twelve receiver locations. These receiver locations include the nine receiver locations of Ionex data (Table 2) along with the three receiver locations of real time TEC data. The reconstruction of the ionosphere with the same geometry (as described in Table 1) is carried out using this new data set. This reconstructed ionosphere is represented as T3 model of the ionosphere in the rest of the paper and is used as background ionosphere for ray tracing at $7.5 \mathrm{MHz}$.

Data set 3: The real time TEC data for three receiver locations collected from the Institute of Radio Physics and Electronics of the University of Calcutta, India for September 1, 2011, at 10 UT are used for reconstruction of the ionosphere with the same geometry (as described in Table 1). This reconstructed ionosphere is represented as T4 model of the ionosphere in the rest of the paper and is used as background ionosphere for ray tracing at $7.5 \mathrm{MHz}$.

The ray tracing results for T2 model ionosphere for Case 1 and Case 2 are shown in Figure 7. The background image of Figure 7 is the reconstructed ionosphere i.e. T2 model of the ionosphere. The ray tracing without the existence of horizontal gradient are shown in Figures $7 \mathrm{a}$ and $7 \mathrm{~b}$. Figures $7 \mathrm{c}$ and $7 \mathrm{~d}$ are the same but under the existence of horizontal gradient. Figures $7 \mathrm{~b}$ and $7 \mathrm{~d}$ are the ray paths under the influence of Earth's magnetic field. Figure 8 represents the ray parameter variations with angle of incidence for T2 and T3 model 


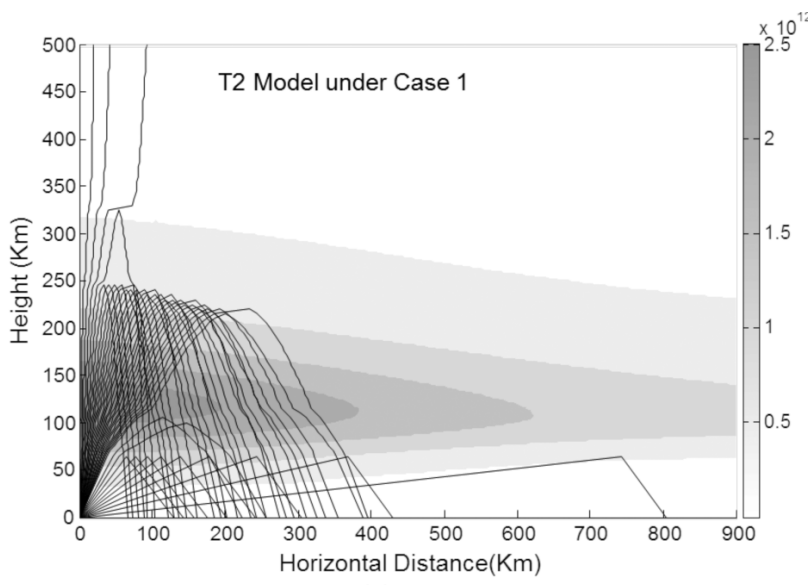

(a)

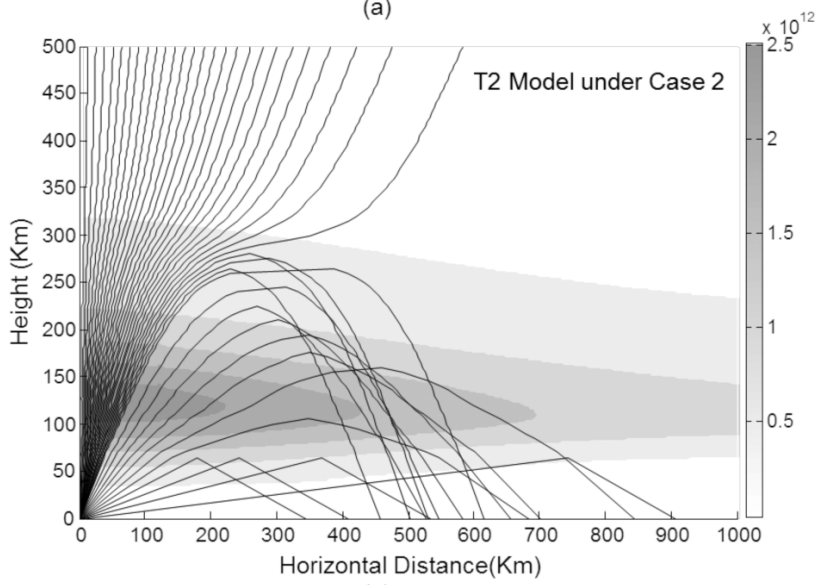

(c)

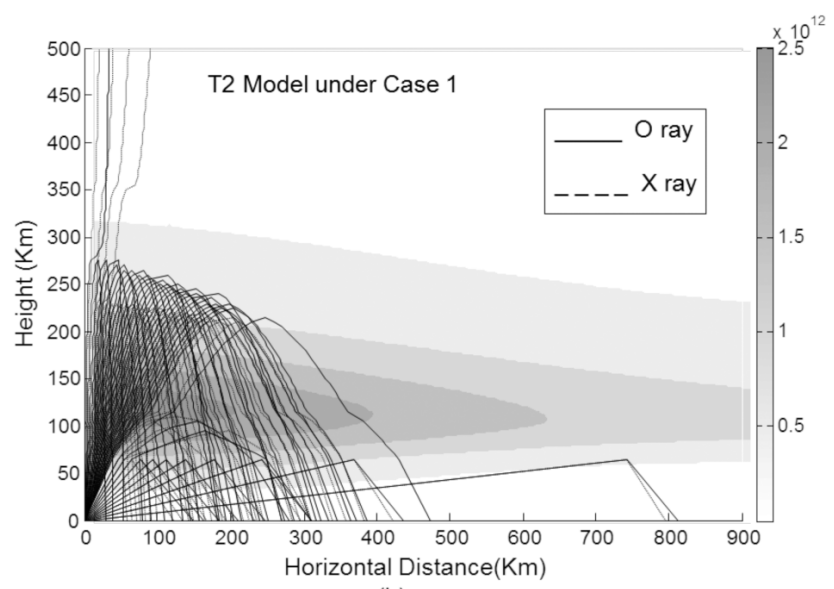

(b)

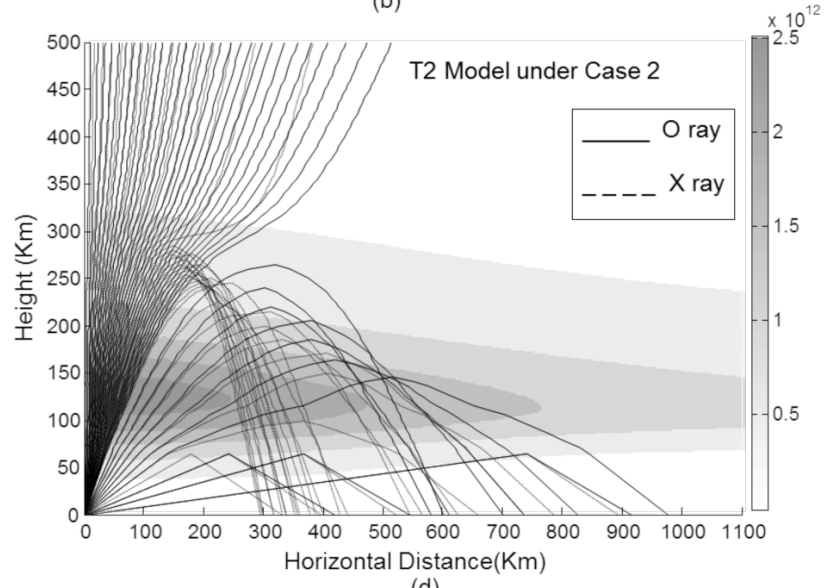

(d)

Figure 7. Ray tracing for different angle of incidence with frequency 7.5 MHz using the T2 model of the ionosphere: (a) without influence of magnetic field under Case 1, (b) with influence of magnetic field under Case 1, (c) without influence of magnetic field under Case 2, (d) with influence of magnetic field under Case 2.

of the ionosphere. The left column of Figure 8 shows the results under Case 1 i.e. under horizontal stratification and the right column shows the results under Case 2 i.e. under horizontal gradient. Ray tracing parameter variation with angle of incidence for T4 model of the ionosphere is shown in Figure 9.

\section{Discussions}

Comparison of ray tracing as described in Section 3 for IRI model of the ionosphere and for two different reconstructions i.e. T1 model and T2 model of the ionosphere reveals good matching of the results. Specifically, the study of Figures 3, 5, and 7 shows that, under the existence of horizontal gradient, the number of reflected rays are less than that for without the gradient. Also Figures $5 \mathrm{~b}, 5 \mathrm{~d}, 7 \mathrm{c}$, and $7 \mathrm{~d}$ establish good matching of the ray tracing considering different ionospheric model. Observation of these figures shows that under horizontal stratification; the reflection heights for different angles of incidence are lower for the case of T2 model while under the existence of horizontal gradient; the reflection heights are larger for the case of T2 model.

The comparison of calculated ray tracing parameters gives expected result that, true path for the ray are larger than the phase path but smaller than the group path. Comparison of Figures 4, 6, and 8 reveals that under horizontal stratification, the values for $\mathrm{P}, \mathrm{P}^{\prime}$, and $\mathrm{D}$ are closer in all the cases. Some numerical instability has been observed in Figures 4 and 6 . This is because; we used iterative method to reconstruct the ionosphere using CIT technique. The accuracy of the method depends on the number of pixels in the grid that are covered by the ray paths. As much number of pixels will be covered by the ray paths, the accuracy of reconstruction will increase. In our iterative reconstruction method, the root mean squared error (RMSE) is nearly $4 \%$ to $8 \%$ depending of the number of receivers used. For this reason, during the tomographic reconstruction process, the pixel values that are not covered by the ray paths are largely differ from the initial guess values whereas the difference of values for the pixels on the ray path are small. Clearly, this difference increases mostly in the lower level of the ionosphere as fewer pixels are covered by the ray paths in the lower regions. Thus the refractive index values for each layer of the ionospheric grid differ from the IRI model and the difference is larger in the lower region. This leads to the numerical differences in the ray parameter calculations in Figure 4. For 


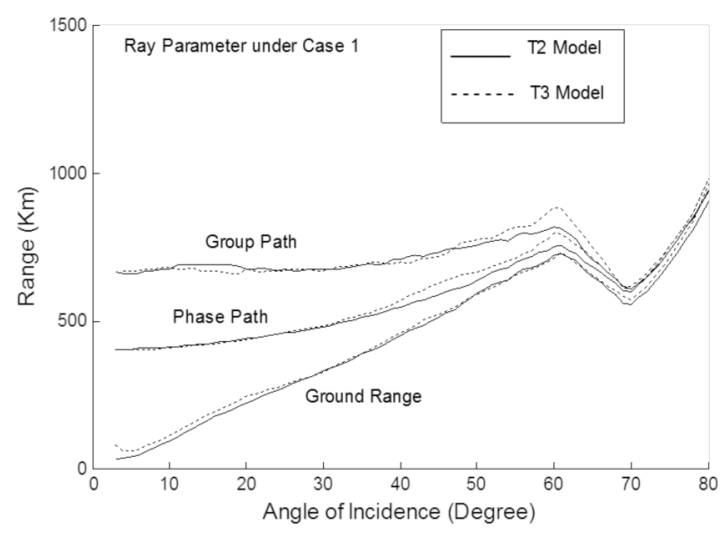

(a)

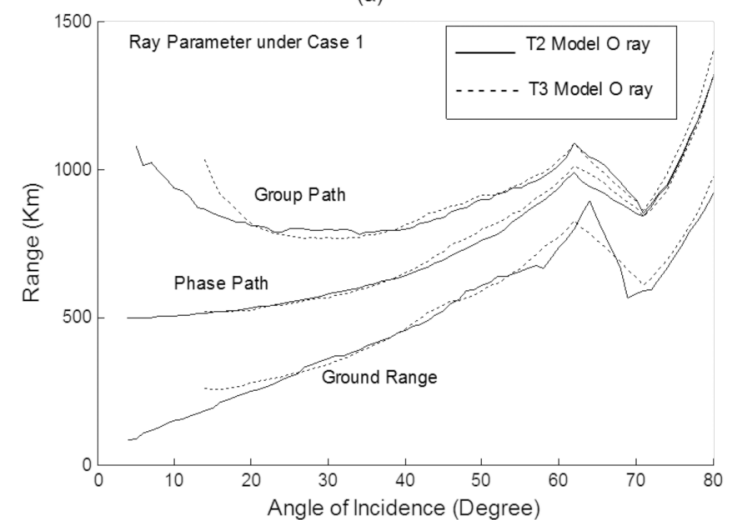

(b)

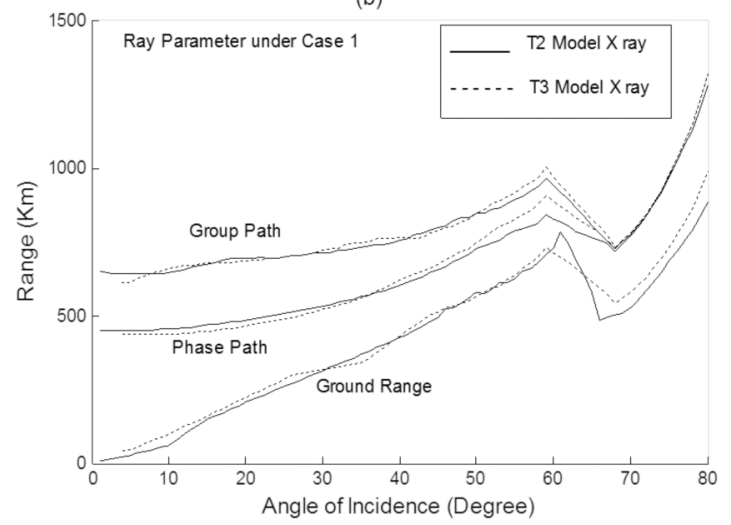

(c)

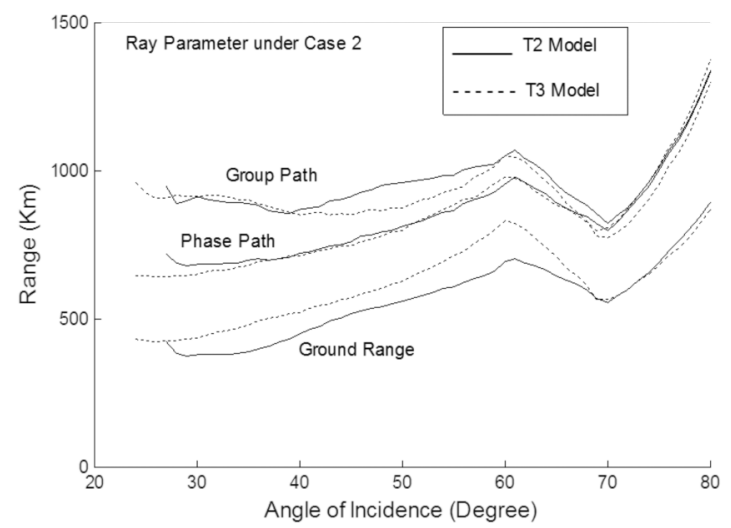

(d)

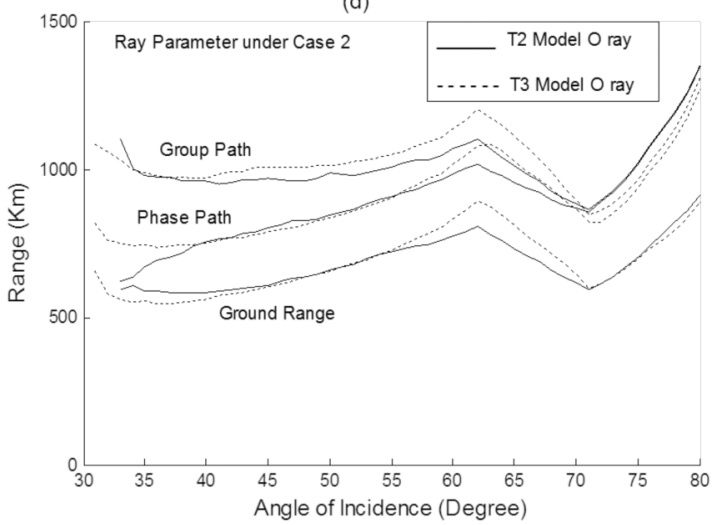

(e)

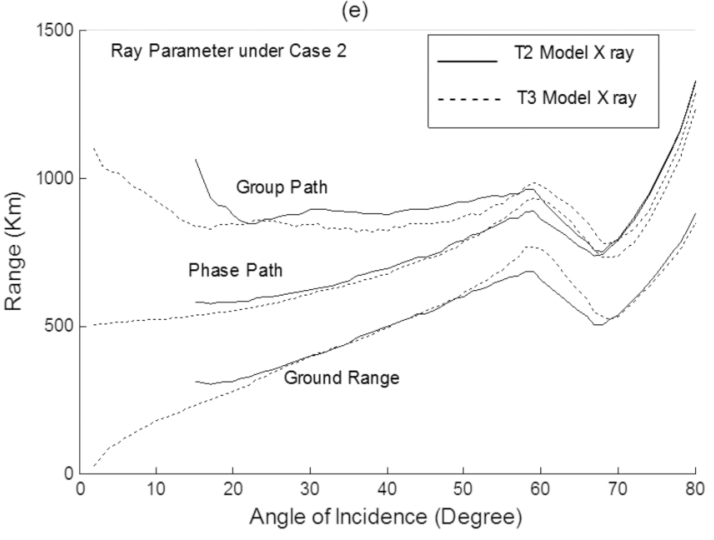

(f)

Figure 8. Ray tracing parameter variation for different angle of incidence at 7.5 $\mathrm{MHz}$ for T2 and T3 model of the ionosphere: (a) without magnetic field under Case 1, (b) O mode under Case 1, (c) X mode under Case 1, (d) without Earth's magnetic field under Case 2, (e) O mode under Case 2, (f) X mode under Case 2.

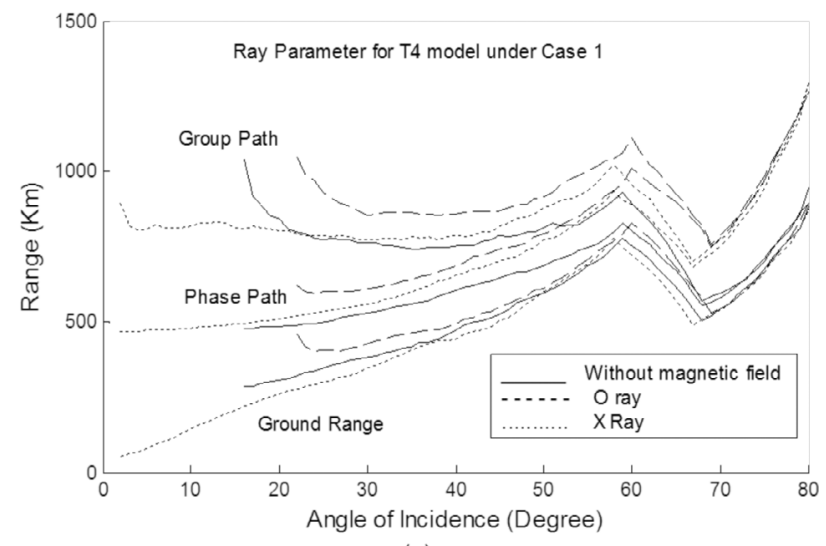

(a)

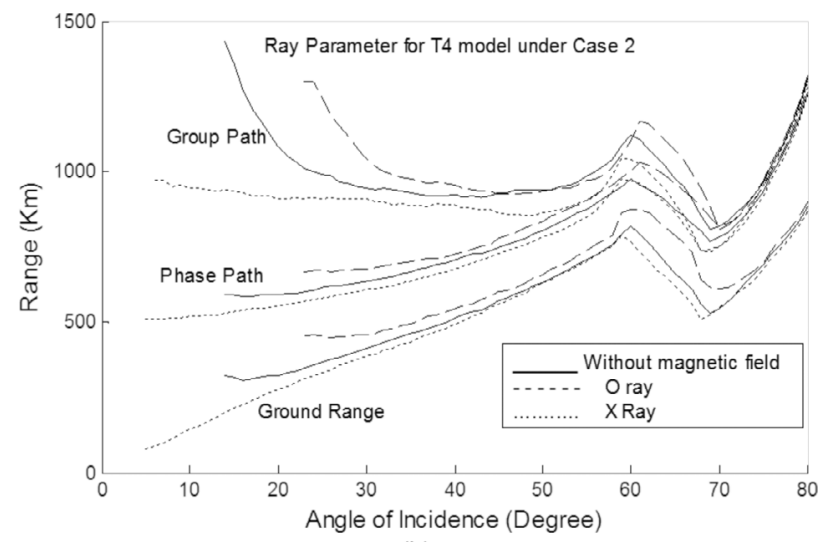

(b)

Figure 9. Ray tracing parameter variation for different angle of incidence at 7.5 MHz for T4 model of the ionosphere: (a) under Case 1 and (b) under Case 2 . 
the case of Figure 6, we considered the effect of horizontal gradient thereby considering the refractive index for each pixel in the grid separately for ray tracing. Obviously the difference in values from initial guess is comparatively smaller from the previous case. Consequently the numerical differences in the ray parameter calculations are less compared to the previous case. Both Figures 4 and 6 show more numerical instability under certain range of angle of incidence. This is because, in that range of incidence angles, the ray trace follows the path through a large number of pixels in the grid out of which maximum number of pixels are uncovered by the ray paths during tomographic reconstruction, thereby increasing the error in ray parameter calculation. In Figure 8, the difference in values for T2 and T3 model of the ionosphere is due to the fact that, more number of receivers will give better reconstruction results with lesser amount of error. Also observation shows the parameter values for O-rays are larger than X-ray's. But an overall result establishes the fact that tomographic reconstruction can be used to develop more general ionospheric model for ray tracing, thus defeating the restriction of the choice of ionospheric model for analytical ray tracing.

For further analysis, ray tracing for fixed angle of incidence is carried out and the minimum frequency for which the ray will escape outwards rather to reflect from the ionosphere are calculated. Since ionosphere with horizontal variation of electron concentration represent a more realistic model, here results only for those rays under the existence of horizontal gradient are produced as shown in Figure 10. Figures 10a and $10 \mathrm{~b}$ are found without and with consideration of magnetic fields respectively. These figures illustrate that as the angle of incidence increases, minimum frequency for escape also increase i.e. higher frequency waves can be reflected from the ionosphere for grazing angle of incidence. Also Figure 10b shows higher probability of reflection of high frequency waves for O-mode compared to X-mode. A comparison of the maximum usable frequency (MUF) for oblique incidence of radio waves has also been shown in Figure 11 for all three models under existence of horizontal gradient. It also shows good matching of the result for IRI, T1, T2, T3 and T4 models. Observation reveals that MUF for oblique incidence is larger than the plasma frequency we choose in this work and that MUF will increase for larger ground range as expected.

Finally the computer run time of the ray tracing algorithm presented in this paper are calculated with different models. The run times are calculated in a Pentium $1 \mathrm{GHz}$ processor and are tabulated in Table 3 for performance comparison. It should be accentuated that

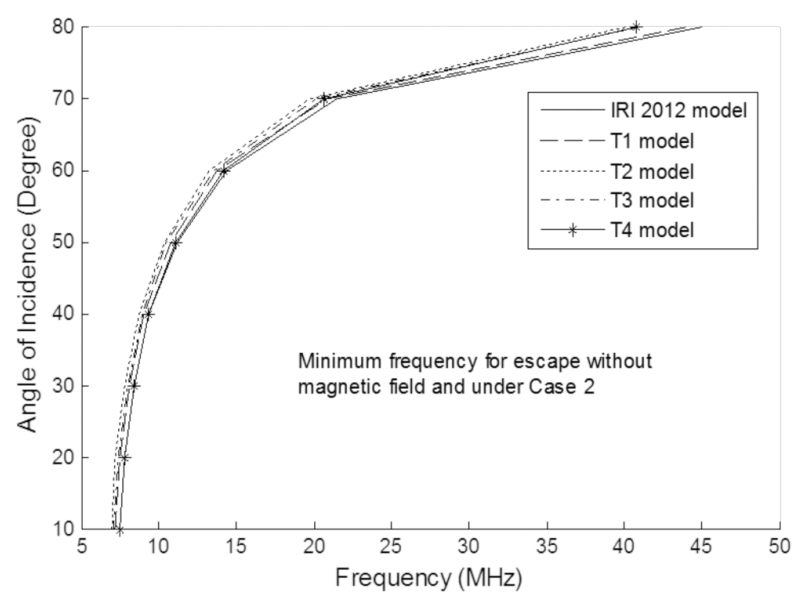

(a)

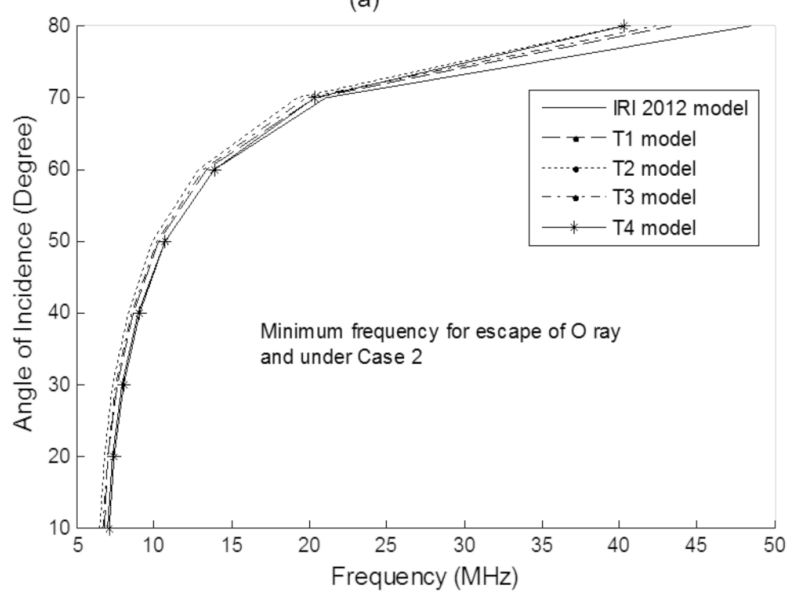

(b)

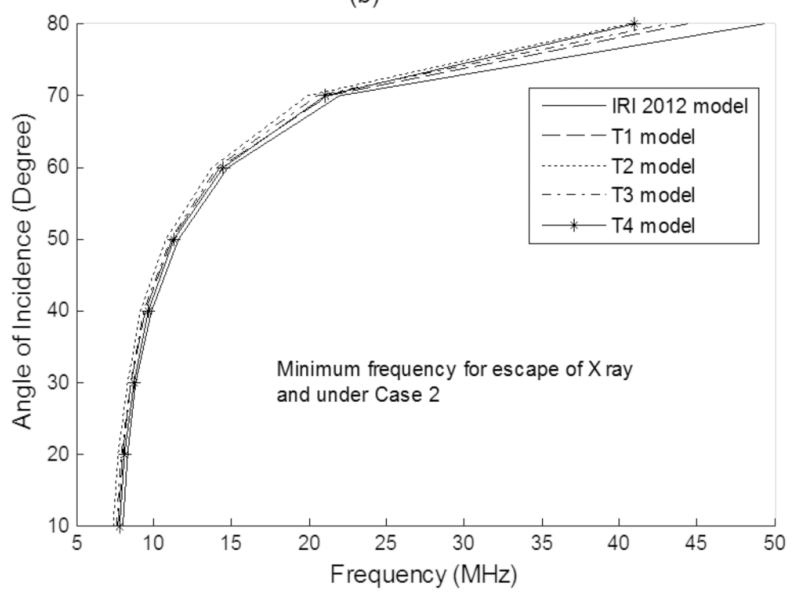

(c)

Figure 10. Variation of minimum frequency for escape with angle of incidence under Case 2 for: (a) without influence of magnetic field, (b) for O mode under influence of magnetic field, (c) for X mode under influence of magnetic field.

no attempt of optimization of the code was made for speed of execution. In Table 3, the ranges are given for the cases whether magnetic field is taken into account or not. Obviously, when magnetic field is introduced, the run time increases slightly. Norman and Cannon [1999] shows the performance of analytical ray tracing methods for different ionospheric model and calculated the speed of execution in a Pentium $100 \mathrm{MHz}$ PC. For comparison purpose, the range of runtime (based on 


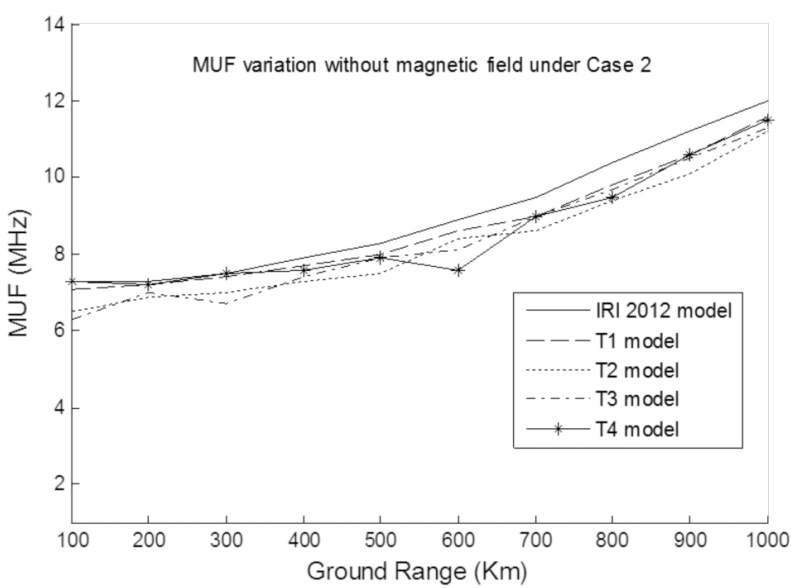

(a)

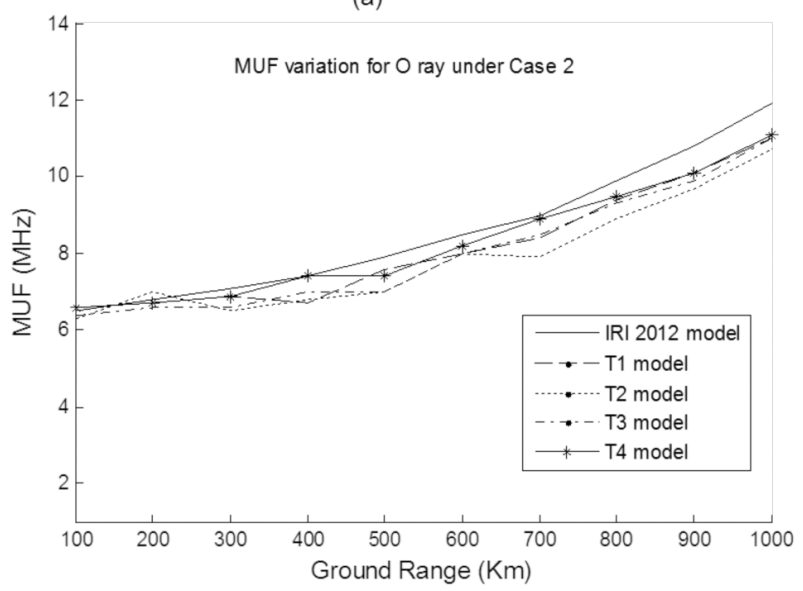

(b)

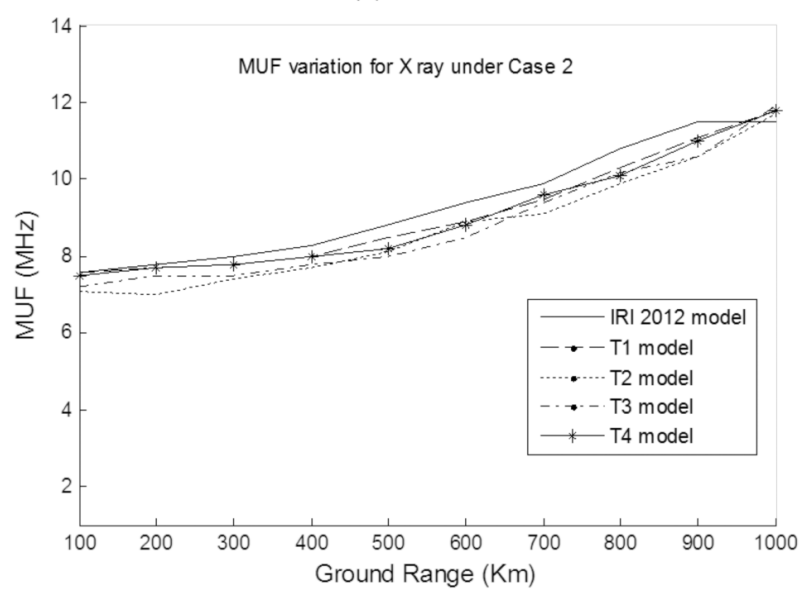

(c)

Figure 11. MUF comparison under Case 2, (a) without influence of magnetic field, (b) for O mode under influence of magnetic field, (c) for $\mathrm{X}$ mode under influence of magnetic field.

their different ionospheric models) for analytical and numerical methods has been quoted from their work in Table 3 as reference. Since higher version of processor is used in the present work, careful observation reveals that execution time of tomographic methods presented in this paper are almost comparable to that of the analytical techniques. Thus, the above discussions on the tomographic ray tracing method along with Table 3 ensures that ionospheric tomography can be used to represent more general and realistic ionospheric models

\begin{tabular}{lc} 
Model for ray tracing & Run time (s) \\
\hline IRI model & $2.4-3.7$ \\
IRI model with horizontal gradient & $8-10.6$ \\
T1 model & $2.4-5.2$ \\
T1 model with horizontal gradient & $6.9-10.7$ \\
T2 model & $2.2-3.4$ \\
T2 model with horizontal gradient & $5.5-10.9$ \\
T3 model & $2.5-5.2$ \\
T3 model with horizontal gradient & $7-10.7$ \\
T4 model & $2.2-3.4$ \\
T4 model with horizontal gradient & $5.5-10.6$ \\
SMART (analytical) & $21-68$ \\
Tilting method 1 (analytical) & $49-62$ \\
Tilting method 2 (analytical) & $49-62$ \\
HIRT (numerical) & $241-640$ \\
\hline
\end{tabular}

Table 3. Performance analysis of different tomographic model and comparison with existing analytical and numerical models.

and an accurate ray tracing can be obtained that is competent with other existing methods.

\section{Conclusion}

In this paper, a ray tracing of HF radio waves has been investigated using more general and realistic ionospheric background. A low latitude ionospheric background has been generated using ionospheric tomography technique based on IRI 2012 model data and also with available GPS - TEC data. The analysis shows accurate ray tracing for HF waves, thus defeating the restriction of choice of ionospheric model for other existing ray tracing methods. Also comparing the results with other existing methods, the efficiency of the ray tracing methods as described in this paper has been ensured.

Acknowledgements. The authors are thankful to the Institute of Radio Physics and Electronics of the University of Calcutta, for providing the measurement data. Authors also thank: NSSDC, USA for providing IRI 2012 model data; NASA, USA for providing Ionex data; and NOAA, National Geophysical Data Centre, for providing Earth's magnetic field data.

\section{References}

Andersen, A.H., and A.C. Kak (1984). Simultaneous algebraic reconstruction technique (SART): a superior implementation of the ART algorithm, Ultrasonic Imaging, 6, 81-94.

Anderson, D.N., J.M. Forbes and M. Codrescu (1989). A 
fully analytic ionospheric model (FAIM), Journal of Geophysical Research, 94, 1520-1524.

Andreeva, E.S., A.V. Galinov, V.E. Kunitsyn, Y.A. Mel'nichenko, E.D. Tereshchenko, M.A. Filimonov and S.M. Chernyakov (1990). Radio tomographic reconstruction of ionization dip in the plasma near the earth, Journal of Experimental and Theoretical Physics Letter, 52, 145-148.

Andreeva, E.S., S.J. Franke, K.C. Yeh and V.E. Kunitsyn (2000). Some features of the Equatorial Anomaly revealed by ionospheric tomography, Geophysics Research Letter, 27, 2465-2468.

Austen, J.R., S.J. Franke, C.H. Liu and K.C. Yeh (1986). Application of Computerized tomography techniques to ionospheric research, In: A. Taurianen (ed.), Radio beacon contribution to the study of ionisation and dynamics of the ionosphere and corrections to geodesy, Oulu, Finland, Part 1, 25-35.

Austen, J.R., S.J. Franke and C.H. Liu (1988). Ionospheric imaging using computerized tomography, Radio Science, 23, 299-307.

Azzarone, A., C. Bianchi, M. Pezzopane, M. Pietrella, C. Scotto and A. Settimi (2012). IONORT: A Windows software tool to calculate the HF ray tracing in the ionosphere, Computers \& Geosciences, 42, 5763; doi:10.1016/j.cageo.2012.02.008.

Bean, B.R., and E.J. Dutton (1966). Radio Metrology, National Bureau of Standards Monograph 92, U.S. Government printing office, Washington.

Bennett, J.A., P.L. Dyson and R.J. Norman (2004). Progress in radio ray tracing in the ionosphere, Radio Science Bulletin, 300, 81-91.

Booker, H.G. (1935). The application of the magnetoionic theory to the ionosphere, Proceedings of Royal Society of London, Ser. A, 150, 267-286.

Budden, K.G. (1952). The theory of the limiting polarization of radio waves reflected from the ionosphere, Proceedings of Royal Society of London, Ser. A, 215-233.

Budden, K.G. (1988). The Propagation of Radio Waves: The Theory of Radio Waves of Low Power in the Ionosphere and Magnetosphere, Cambridge University Press, Cambridge, UK, 688.

Coleman, C.J. (1998). A ray-tracing formulation and its application to some problems in over-the horizon radar, Radio Science, 33 (4), 1187-1197; doi:10.1029/ 98RS01523.

Davies, K. (1990). Ionospheric Radio, Peter Peregrinus Ltd. (ed.) on behalf of the Institution of Electrical Engineers (IET), London, UK, 508.

Försterling, K. (1942). Über die Ausbreitung elektromagnetischer Wellen in einem magnetisierten Medium beisenkrechter Inzidenz, Hochfrequen- ztech Elektroakust., 59, 10-22.

Haselgrove, J. (1955). Ray theory and a new method of ray tracing, Conference on the Physics of the Ionosphere, Proceedings of the Physical Society of London, 23, 355-364.

Hines, C.O. (1951). Wave packets, the Poyiting vector, and energy flow: 1 . Nondispersive (anisotropic) homogeneous media, Journal of Geophysical Research, $56,63-72$.

Huang, C.S., G. Sofko and M. Kelley (1998). Numerical simulations of midlatitude ionospheric perturbations produced by gravity waves, Journal of Geophysical Research, 103, 6977-6989.

Jakowski, N., E. Sardon, E. Engler, A. Jungstand and D. Klahn (1996). Relationship between GPS-signal propagation errors and EISCAT observations, Annales Geophysicae, 14, 1429-1436.

Jones, R.M. (1966). A three dimensional ray tracing computer program, ESSA Technical Report, IER 17ITS, 17.

Jones, R.M., and J.J. Stephenson (1975). A versatile threedimensional ray tracing computer program for radio waves in the ionosphere, OT Report, 75-76, U.S. Department of Commerce, Office of Telecommunication, U.S. Government Printing Office, Washington, USA, 185.

Kersley, L., S.E. Pryse, I.K. Walker, J.A.T. Heaton, C.N. Mitchell, M.J. Williams and C.A. Willson (1997). Imaging of electron density troughs by tomographic techniques, Radio Science, 32, 1607-1621.

Kunitake, M., K. Ohtaka, T. Maruyama, M. Tokumaru, A. Morioka and S. Watanabe (1995). Tomographic imaging of the ionosphere over Japan by the modified truncated SVD method, Annales Geophysicae, 13, 1303-1310.

Kunitsyn, E.V., and E.D. Tereshchenko (1992). Radio tomography of the ionosphere, IEEE Antennas and Propagation Magazine, 34, 22-32.

Leitinger, R. (1999). Ionospheric tomography, In: Review of Radio Science 1, 1996-1999, edited by W. R. Stone, Oxford Univ. Press, New York, chap. 24, pp. 581-623.

Markkanen, M., M. Lehtinen, T. Nygren, J. Pirttila, P. Henelius, E. Vilenius, E.D. Tereshchenko and B.Z. Lhuduk (1995). Bayesian approach to satellite radio tomography with applications in the Scandinavian sector, Annales Geophysicae, 13, 1277-1287.

Mitchell, C.N., D.G. Jones, L. Kersley, S.E. Pryse and L.K. Walker (1995). Imaging of field-aligned structures in the auroral ionosphere, Annales Geophysicae, 13, 1311-1319.

Nandi, S., and B. Bandyopadhyay (2015). Study of lowlatitude ionosphere over Indian region using simul- 
taneous algebraic reconstruction technique, Advances in Space Research, 55, 545-553.

Nickish, L.J. (2008). Practical application of Haselgrove's equation for HF systems, Radio Scientific Bulletin URSI N. 325, 36-48.

Norman, R.J., and P.S. Cannon (1997). A two dimensional analytic ray tracing technique accommodating horizontal gradients, Radio Science, 32, 387-396.

Norman, R.J., and P.S. Cannon (1999). An evaluation of a new two-dimensional analytic ionospheric raytracing technique (SMART), Radio Science, 34, 489499.

Poeverline, H. (1948). Strahlwege von Radiowellen in der Ionosphere (I), Sitzungsberichte der Bayerischen Akademie der Wissenschsften, 175-201.

Poeverline, H. (1949). Strahlwege von Radiowellen in der Ionosphere (II), Zeitschrift fur angewandtte Physik, 517-525.

Pryse, S.E., C.N. Mitchell, J.A.T. Heaton and L. Kersley (1995). Traveling ionospheric disturbances imaged by tomographic techniques, Annales Geophysicae, 13, 1325-1342.

Pryse, S.E., L. Kersley, C.N. Mitchell, P.S.J. Spencer and M.J. Williams (1998). A comparison of reconstruction techniques used in ionospheric tomography, Radio Science, 33, 1767-1779.

Raymund, T.D. (1995). Comparison of several ionospheric tomography algorithms, Annales Geophysicae, 13, 1254-1262.

Reilly, M.H. (1991). Upgrades for efficient three dimensional ionospheric ray tracing: Investigation of HF near vertical incidence sky wave effects, Radio Science, 26, 971-980.

Rydbeck, O.E.H. (1944). On the propagation of radio waves, Trans. Chalmers Univ. Technol., 34, 1-170.

Settimi, A., M. Pezzopane, M. Pietrella, C. Bianchi, C. Scotto, E. Zuccheretti and J. Makris (2013). Testing the IONORT-ISP system: a comparison between synthesized and measured oblique ionograms, Radio Science, 48 (2), 167-179; doi:10.1002/rds.20018.

Yeh, K.C., E.S. Andreeva, S.J. Franke and V.E. Kunitsyn (2001). An investigation of motions of the equatorial anomaly crest, Geophysics Research Letter, 28, 4517-4520.

\footnotetext{
${ }^{\star}$ Corresponding author: Sandip Nandi, Kalyani Govt. Engineering College, Department of Electronics and Communication Engineering, Kalyani, West Bengal, India; email: sandip622@yahoo.co.uk. 